\title{
Acumulação de metais em sedimentos de área de captação para abastecimento no Sul do Brasil
}

Os metais pesados têm se tornado uma problemática, devido a sua capacidade de em contato com seres vivos desenvolver processos de bioacumulação e biomagnificação, bem como, processos de poluição e/ou contaminação pontual e difusa. No entanto, os elementos químicos que compõe a classes dos metais pesados, em pequenas quantidades são necessárias ao bom funcionamento dos organismos vivos, bem como, os mesmos apresentam-se como componentes naturais de rochas e solos, que em processos de dissolução tornam-se disponíveis ao ambiente, estando no solo e na água. Dessa forma, o presente estudo objetiva avaliar se os teores elevados de Fe e Mn dissolvidos na água da Bacia Hidrográfica do Epaminondas, no município de Pelotas/RS. Para isso, foram utilizados índices ambientais que indicam se os teores observados são provenientes de fonte natural ou antrópica, e se estes teores já provocam a contaminação do ambiente em estudo. Foram realizadas coletas de sedimentos em suspensão e de fundo na bacia em estudo, em cinco pontos de coleta e nestes sedimentos procedeu-se caracterização química e a partir dos teores de $\mathrm{Cu}$, Fe, $\mathrm{Mn}$ e Zn elaborou-se o Índice de Geoacumulação, Fator de Enriquecimento, Fator de Contaminação, Grau de Contaminação, Grau de Contaminação modificado e Índice de Carga Poluidora. Após a elaboração dos índices propostos, observou-se que os teores encontrados ainda não são considerados contaminantes e que os elementos dissolvidos provêm de fonte natural.

Palavras-chave: Índices ambientais; Contaminantes ambientais; Bacias Hidrográficas.

\section{Accumulation of metals in sediments from a water catchment area for supply in Southern Brazil}

\begin{abstract}
Heavy metals are more often becoming a problem to living beings due to their ability to bind organically in processes of bioaccumulation and biomagnification, as well to the natural environment where it can cause pollution and punctual or diffuse contamination. However, chemical elements that compound the heavy metal class are essential in small quantities for living organisms since they are natural components that are also contained in rocks and soils, which after dissolution processes may decay and be available in the ground and water. Therefore, the present study aimed to evaluate the levels of Fe and Mn dissolved in the water of the Epaminondas Hydrographic Basin, located in the municipality of Pelotas, RS. Environmental indexes were used to indicate whether the observed levels are from a natural or anthropic source and whether these levels are already contaminating the environment. Suspended and bottom sediments samples were collected from the basin at five different locations. The chemical description of these sediments was carried out based on their $\mathrm{Cu}$, Fe, Mn, and $\mathrm{Zn}$ contents, Geoaccumulation Index, Factor of Enrichment, Contamination Factor, Degree of Contamination, Modified Degree of Contamination, and Pollutant Load Index. After elaborating on the proposed indexes, the heavy metal levels were deemed non-contaminant elements, classified as originated from a natural source.
\end{abstract}

Keywords: Environmental indexes; Environmental contaminants; Drainage Wathersheds.

Topic: Uso de Recursos Naturais

Reviewed anonymously in the process of blind peer
Received: 08/06/2021

Approved: 27/06/2021
Eliana Aparecida Cadoná (iD)

Universidade Federal de Pelotas, Brasil http://lattes.cnpq.br/8511698054229545 http://orcid.org/0000-0003-4556-4259 elianacadona@yahoo.com.br

Stefan Domingues Nachtigall (D) Universidade Federal de Pelotas, Brasil http://lattes.cnpq.br/8700561006180244 http://orcid.org/0000-0003-4623-8033 stefan.tefo@gmail.com

Jéferson Diego Leidemer (iD)

Universidade Federal de Pelotas, Brasil http://lattes.cnpq.br/3015484877843039 http://orcid.org/0000-0002-6727-9953 jeferson.leidemer@gmail.com

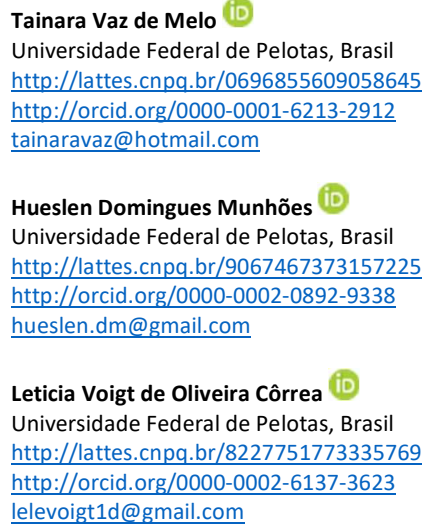

Beatriz Bruno do Nascimento (D)

Universidade Federal de Pelotas, Brasil http://lattes.cnpq.br/2386715472776988 http://orcid.org/0000-0002-9524-6067 beatrizncmbruno@gmail.com

Pablo Miguel (iD)

Universidade Federal de Pelotas, Brasil http://lattes.cnpq.br/4145554276881172 http://orcid.org/0000-0002-7999-8912 pablo.ufsm@gmail.com MUNHÕES, H. D.; CÔRREA, L. V. O.; NASCIMENTO, B. B.; MIGUEL, P.. Acumulação de metais em sedimentos de área de captação para abastecimento no Sul do Brasil. Revista Ibero Americana de Ciências Ambientais, v.12, n.6, p.615-624, 2021. DOI: http://doi.org/10.6008/CBPC2179-6858.2021.006.0051 


\section{INTRODUÇÃO}

A dinâmica de metais pesados tem sido utilizada em estudos ambientais, em especial naqueles de monitoramento da qualidade da água, pois são elementos biologicamente essenciais em pequenas quantidades para os processos fisiológicos e bioquímicos (MATOS et al., 2017). Esses metais pesados, quando ultrapassam os limites da essencialidade no ambiente, acabam por comprometer a qualidade da água e do solo, bem como interferir nos processos de equilíbrio ambiental (GONÇALVES et al., 2015). Para o ser humano, de maneira geral, os metais pesados são necessários em pequenas quantidades para o bom funcionamento do organismo (MATOS et al., 2017). Quando presentes em quantidades acima das necessárias ao organismo, podem ocasionar quadros stress oxidativo aos tecidos, podendo levar a diversas doenças, como inflamações em tecidos, lesões neurológicas, lesões em nível de DNA, no metabolismo da glicose, bem como a indução a processos mutagênicos e carcinogênicos (KORASHY et al., 2017). Dessa maneira, a presença de metais pesados no solo, na água ou nos sedimentos pode ocasionar riscos as populações abastecidas, sendo necessário, o estudo da dinâmica dos mesmos no ambiente, em especial nos sedimentos, levando em consideração que problemas ambientais envolvendo metais pesados são comuns.

A presença de metais pesados em sedimentos pode ser identifica por meio do Índice de Geoacumulação - Igeo (MÜLLER, 1979). O Igeo constitui na atribuição de valores e de graus de contaminação por metais pesados em sedimentos e tem sido utilizado no Brasil, em regiões estuarinas e de grande concentração industrial, para avaliar se a contaminação dos sedimentos é proveniente de fontes antrópicas ou naturais. No entanto, esse índice também pode ser aplicado para estudos de regiões afastadas de centros urbanos desde que haja a premissa de altos índices de contaminação nas águas de reservatórios.

Nesse sentido, Oliveira et al. (2015) avaliaram os teores de $\mathrm{Cr}, \mathrm{Cu}, \mathrm{Ni}, \mathrm{Pb}$ e $\mathrm{Zn}$ nos sedimentos de fundo da baia do rio Guajará e rio Carnapijó. Ambas as bacias afastadas da cidade de Belém/PA, e observaram que, a aplicação do Igeo indicou que o $\mathrm{Pb}$ foi o metal que apresentou maiores valores nos sedimentos e que o mesmo foi carreado por distâncias maiores que os demais elementos analisados.

A aplicação do Igeo também tem sido realizada com foco no $\mathrm{Hg}$ em áreas de grande concentração industrial, devido a elevada periculosidade ambiental que o mesmo apresenta. Em estudo realizado em ambientes não degradados das nascentes da Bacia Hidrográfica dos Educandos, no município de Manaus, estado do Amazonas, Torrezani et al. (2017) compararam concentrações de $\mathrm{Hg}$ com valores naturais e a partir destes compararam com os valores de Igeo, utilizando nove pontos amostrais. $O$ estudo mostrou que, nos pontos amostrais, somente um foi classificado como pouco a moderadamente contaminado, indicando assim que a origem da contaminação não é natural, ou seja, oriunda da grande concentração de indústrias na região.

No Sul da China, Xiao et al. (2019), utilizaram o Índice de Geoacumulação - Igeo e o risco potencial ecológico nos sedimentos para observar a distribuição espacial e temporal dos metais $\mathrm{Cd}, \mathrm{Pb}, \mathrm{Mn}, \mathrm{Zn}, \mathrm{Cu}, \mathrm{Ni}$, $\mathrm{Cr}$, As e $\mathrm{Hg}$, associado à sua correlação no ambiente. Os autores observaram que o $\mathrm{Mn}$ é o metal que apresentou as maiores concentrações e espacialmente os metais encontravam-se na classe 3 do Índice de 
Geoacumulação, ao qual indicam sedimentos moderadamente a fortemente contaminados. Na Região do North Al-Waih, Mar Vermelho na Arábia Saudita, Youssef et al. (2020) avaliaram a concentração dos metais $\mathrm{Al}, \mathrm{Cd}, \mathrm{Co}, \mathrm{Sr}, \mathrm{V}, \mathrm{Cu}, \mathrm{Fe}, \mathrm{Mn}, \mathrm{Ni}, \mathrm{Pb}$ e Hg em colunas de sedimentos de $200 \mathrm{~cm}$. O Al foi o metal que apresentou as maiores concentrações e consequentemente os maiores índices de geoacumulação, no entanto, os metais $\mathrm{Sr}$ e $\mathrm{Cd}$ foram os que mais enriqueceram os sedimentos de fundo através de atividades antrópicas.

A bacia hidrográfica do presente estudo contribui para o abastecimento de água do município de Pelotas/RS a qual apresenta problemática de teores dissolvidos elevados de Fe e Mn (KATH et al., 2017), o que pode ocasionar danos à saúde das populações abastecidas e elevação nos custos de tratamento da água para o abastecimento humano. Por este motivo surge a necessidade de avaliar o grau de contaminação dos sedimentos de fundo da Bacia Hidrográfica do Epaminondas (BHE), utilizando o Índice de Geoacumulação Igeo e determinar se a possível fonte de contaminação é originária do solo ou antrópica.

\section{MATERIAIS E MÉTODOS}

O estudo foi realizado na Bacia Hidrográfica do Epaminondas (BHE) que está localizada no município de Pelotas, na Região Sul do Estado do Rio Grande do Sul - Brasil. A BHE conta com área de aproximadamente $33,3 \mathrm{~km}^{2}$ (Figura 1) e corresponde a aproximadamente 70\% da Bacia Hidrográfica do Arroio Santa Bárbara, que abastece o município de Pelotas através da Barragem do Santa Bárbara. O clima do local é do tipo Cfa, segundo a classificação de Köeppen, com temperaturas médias em $18,4 \stackrel{\circ}{ } \mathrm{C}$, com precipitação entorno dos 1393,9 mm (KLUMB et al., 2018). O período de estudo correspondeu entre os meses de julho/2018 a dezembro/2019.

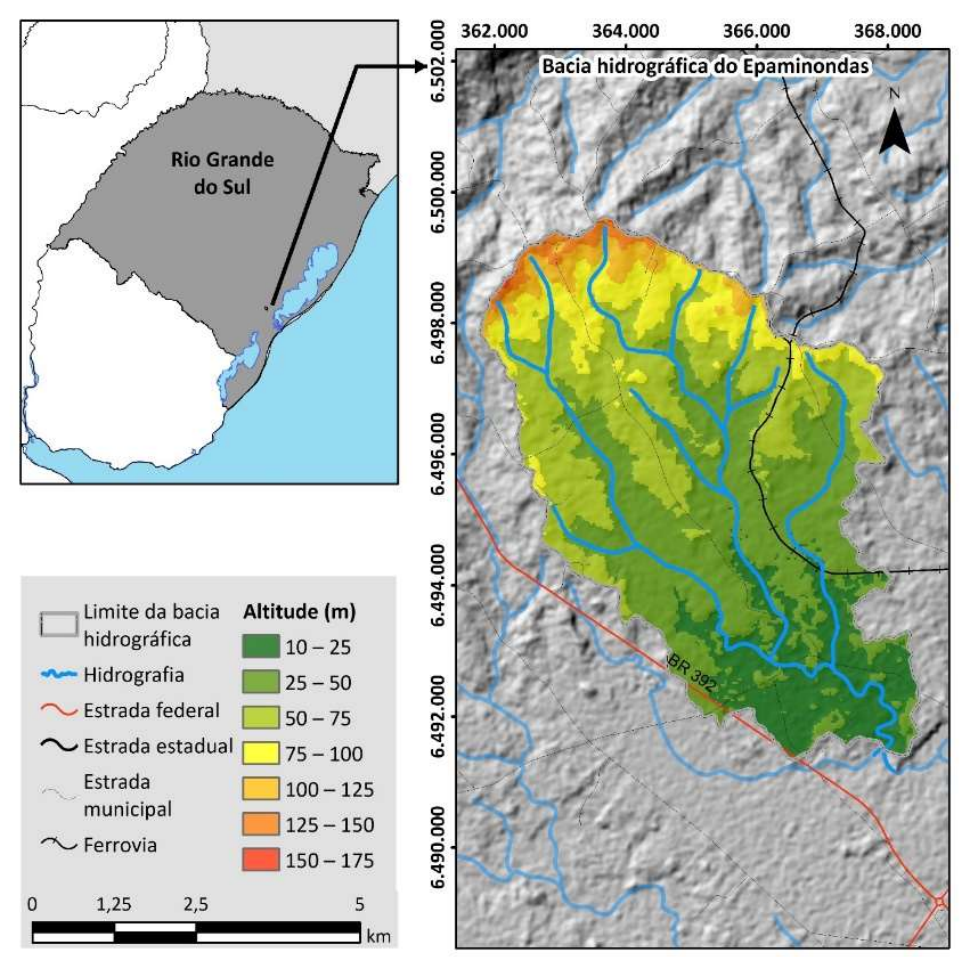

Figura 1:1 Localização geográfica em nível estadual e variação altimétrica da Bacia Hidrográfica do Epaminondas.

Foram realizadas coletas de sedimentos de fundo, no leito das drenagens, através da retirada de 
material depositado com equipamento próprio para esse procedimento, em cinco pontos localizados dentro da BHE (Figura 2a). Dois pontos de coleta na porção Norte da bacia (S1 e S2), dois pontos na porção Sul da bacia (S3 e S4) e um ponto próximo ao exutório. Os locais de coleta foram determinados a fim de representa a maior variabilidade ambiental dentro da área, como por exemplo, a declividade (Figura 2b), os tipos de solos (Figura 2c) e o uso da terra (Figura 2d). As coletas de sedimentos ocorreram nos meses de Março, Junho, Agosto e Dezembro do ano de 2019.

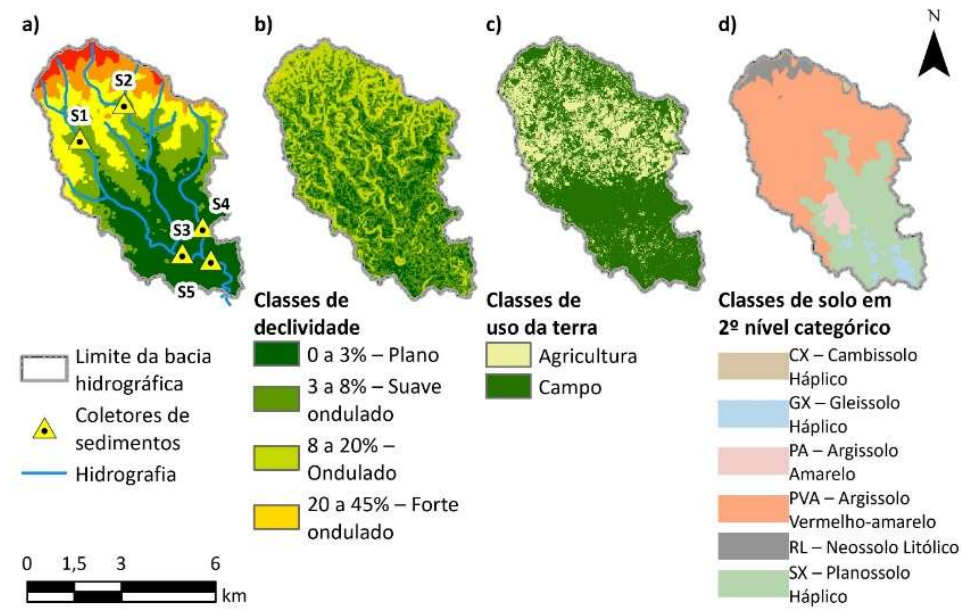

Figura 2: Mapa de distribuição dos coletores de sedimentos (a), das declividades (b), uso da terra (c) e tipos de solos (d) na Bacia Hidrográfica do Epaminondas.

Nos sedimentos coletados foi realizada uma caracterização química através da digestão ácida nitroperclórica (3:1) conforme descrito por Silva (1999) e após esse procedimento, foi determinado, via extrato da digestão, os teores de $\mathrm{Al}, \mathrm{Ca}, \mathrm{Mg}, \mathrm{P}, \mathrm{K}, \mathrm{Na}, \mathrm{Cu}, \mathrm{Zn}$, Fe e $\mathrm{Mn}$. A determinação dos teores de Carbono Orgânico Total (COT) foram determinados por digestão sulfocrômica conforme descrito por Teixeira et al. (2017) e a composição granulométrica foi determinada pelo método da pipeta, conforme preconizado por Teixeira et al. (2017). As concentrações médias de cada análise química $\left(\mathrm{mg} \mathrm{kg}^{-1}\right)$ e a quantidade de argila (\%) encontram-se apresentadas na tabela 1. A partir dos teores dos metais pesados $\mathrm{Al}, \mathrm{Cu}, \mathrm{Fe}, \mathrm{Mn}$ e $\mathrm{Zn}$ encontrados nos sedimentos coletados, foram elaborados o Índice de Geoacumulação (Igeo), Fator de Enriquecimento (FE), Fator de Contaminação (FC), Grau de Contaminação (GC), Grau de Contaminação modificado (GCm) e Índice de Carga Poluidora (ICP).

Tabela 1: Teores de argila (\%) e teores médios das variáveis químicas analisadas $\left(\mathrm{mg} \mathrm{kg}^{-1}\right)$ para sedimentos de fundo (S1, S2, S3, S4 e S5) na Bacia Hidrográfica do Epaminondas no período de estudo.

\begin{tabular}{|c|c|c|c|c|c|c|c|c|c|c|c|c|}
\hline \multirow{2}{*}{ Ponto } & \multirow{2}{*}{ Argila - \% } & \multicolumn{11}{|c|}{ Variável química - $\mathrm{mg} \mathrm{kg}^{-1}$} \\
\hline & & $\mathrm{Al}$ & $\mathrm{Ca}$ & $\mathrm{Cu}$ & $\mathrm{Fe}$ & $\mathrm{K}$ & $\mathrm{Mg}$ & $\mathrm{Mn}$ & $\mathrm{Na}$ & $P$ & $\mathrm{Zn}$ & $\mathrm{MO}$ \\
\hline S1 & 1,12 & 0,90 & 7,89 & 0,05 & 1,17 & 244,38 & 5,40 & 1,01 & 42,09 & 30,99 & 0,43 & 6,80 \\
\hline S2 & 1,00 & 0,77 & 7,19 & 0,19 & 1,29 & 487,35 & 3,21 & 1,36 & 44,54 & 62,46 & 1,39 & 7,01 \\
\hline S3 & 1,00 & 0,75 & 2,33 & 0,08 & 0,60 & 29,90 & 3,57 & 1,20 & 33,21 & 16,29 & 0,29 & 7,63 \\
\hline S4 & 0,59 & 0,74 & 1,47 & 0,01 & 0,39 & 24,38 & 0,59 & 0,41 & 37,30 & 19,66 & 1,27 & 9,06 \\
\hline S5 & 1,00 & 0,87 & 3,74 & 1,10 & 0,56 & 23,11 & 3,56 & 0,90 & 34,25 & 19,94 & 2,62 & 6,76 \\
\hline
\end{tabular}

Para a obtenção do Igeo foi aplicada a equação proposta por Muller (1979) onde: Igeo = $\log 2\left(\frac{C n}{1,5 * C b}\right)$, neste $\mathrm{Cn}=$ concentração do metal medido na amostra; $\mathrm{Cb}=$ concentração de base ou de 
referência e 1,5 = valor que minimiza as variações do valor de referência. Além da classificação pelo Igeo, são obtidos os dois fatores, o Fator de Enriquecimento (FE) e o Fator de Contaminação (FC). O FE foi obtido a partir da aplicação da equação $F E=\frac{C n / A l b}{C b / A l n}$, onde: $C n=$ concentração do metal; Alb: concentração de Al na amostra; Cb e Aln: concentração de referência do metal e de Alumínio e o $\mathrm{FC}$ a partir da equação $F C=\frac{M e a m}{M e b g}$, onde: Meam = concentração do metal no sedimento; Mebg = nível natural do metal ou o seu background. Para o cálculo do Grau de Contaminação (GC) foi utilizada a equação $G C=\sum_{i=l}^{n=4} C F i$, onde soma dos fatores de contaminação dos elementos analisados. Para o Grau de Contaminação modificado $(\mathrm{GCm})$ utilizou-se a equação $G C m=\frac{\left(\sum_{i=l}^{n=4} \mathrm{CFi}\right)}{n}$, onde soma dos fatores de contaminação dos elementos analisados; $\mathrm{n}=$ número de amostras e para o Índice de Carga Poluidora (ICP) utilizou-se a equação ICP $=$ $\sqrt[4]{F C F e * F C M n * F C C u * F C Z n}$, onde $\mathrm{FC}_{\mathrm{Fe}}=$ fator de contaminação para $\mathrm{Fe} ; \mathrm{FC}_{\mathrm{Mn}}=$ fator de contaminação $\mathrm{Mn} ; \mathrm{FCCu}=$ fator de contaminação $\mathrm{Cu} ; \mathrm{FCZn}$ = fator de contaminação Zn. Todos os índices aplicados são interpretados conforme seus valores e enquadrados em classes de qualidades, as quais estão apresentadas na Tabela 2.

Para o cálculo do FE é desenvolvido com a utilização de um metal normalizador, encontrado em teores naturais no solo, podendo ser Fe, Mn ou Al (ABRAHIM et al., 2008). O presente estudo utilizou o Al como metal normalizador, devido a sua presença natural nos solos, oriundo de minerais primários e óxidos de Al e a partir deste cálculo é capaz de se constatar se a origem dos metais nos sedimentos analisados, decorre de fontes naturais ou de fontes antrópicas. Para o FC, infere-se o grau de contaminação dos sedimentos a partir de uma relação entre as concentrações encontradas e as concentrações naturais ou backgrounds do metal em estudo para sedimentos, mostrando que processos de contaminação são mais dificilmente revertidos devido à alta concentração do contaminante no meio analisado.

Tabela 2: Valores dos índices de qualidade de sedimentos (Igeo, FE, FC, GC e GCm) e respectivas classes de qualidade.

\begin{tabular}{lll}
\hline Índice de Geoacumulação & & Classes \\
\hline Igeo & Qualidade & 0 \\
$<0$ & Não contaminado & 1 \\
$0-1$ & Não contaminado a moderadamente contaminado & 2 \\
$1-2$ & Moderadamente contaminado & 3 \\
$2-3$ & Moderadamente a forte contaminado & 4 \\
$3-4$ & Fortemente contaminado & 5 \\
$4-5$ & Fortemente a extremamente contaminado & 6 \\
$>5$ & Extremamente contaminado & \\
\hline Fator de Enriquecimento & \\
\hline FE & & \\
$<1,5-2$ & Origem & \\
$>2$ & Natural & \\
\hline Fator de Contaminação & Antrópica & \\
\hline FC & & \\
$<1$ & Classes & \\
$1-3$ & Contaminação Baixa & \\
$3-6$ & Contaminação Moderada & \\
$>6$ & Contaminação Considerável & \\
\hline Grau de Contaminação - GC & Contaminação Elevada & \\
\hline GC & & \\
$<6$ & Classe de Qualidade \\
$6-12$ & Grau Baixo & \\
\hline
\end{tabular}




\begin{tabular}{ll}
$12-24$ & Grau Considerável \\
$>24$ & Grau Muito Alto \\
\hline Grau de Contaminação modificado - GCm & \\
\hline GCm & Classe de Qualidade \\
$<1,5$ & Nulo a Muito Baixo \\
$1,5-2$ & Baixo \\
$2-4$ & Moderado \\
$4-8$ & Alto grau \\
$8-16$ & Muito Alto \\
$16-32$ & Extremamente Alto \\
$>32$ & Ultra-Alto \\
\hline
\end{tabular}

\section{RESULTADOS}

Para os pontos de estudo da Bacia Hidrográfica do Epaminondas (BHE) durante o período de estudo a concentração média foi de $0,06 \mathrm{mg} \mathrm{kg}^{-1} \mathrm{de} \mathrm{Cu}, 0,67 \mathrm{mg} \mathrm{kg}^{-1}$ de Fe, 0,81 $\mathrm{mg} \mathrm{kg}^{-1}$ de $\mathrm{Mn}$ e de $0,75 \mathrm{mg} \mathrm{kg}^{-1} \mathrm{de}$ $\mathrm{Zn}$ nos sedimentos de fundo, conforme apresentado na figura 3. Observa-se que para os metais $\mathrm{Cu}$ e $\mathrm{Mn}$ ocorreram concentrações de $0,00 \mathrm{mg} \mathrm{kg}^{-1}$ para as coletas 1 e 3, possivelmente em decorrência da baixa concentração nos sedimentos, fazendo com que o aparelho de absorção atômica não consiga realizar a detecção após as análises químicas. A aplicação do Igeo resultou em valores <0 (Figura 4) para todos os pontos e coletas, enquadrando-os como classe nula de contaminação indicando que os sedimentos analisados não se encontram contaminados pelos metais em estudo.
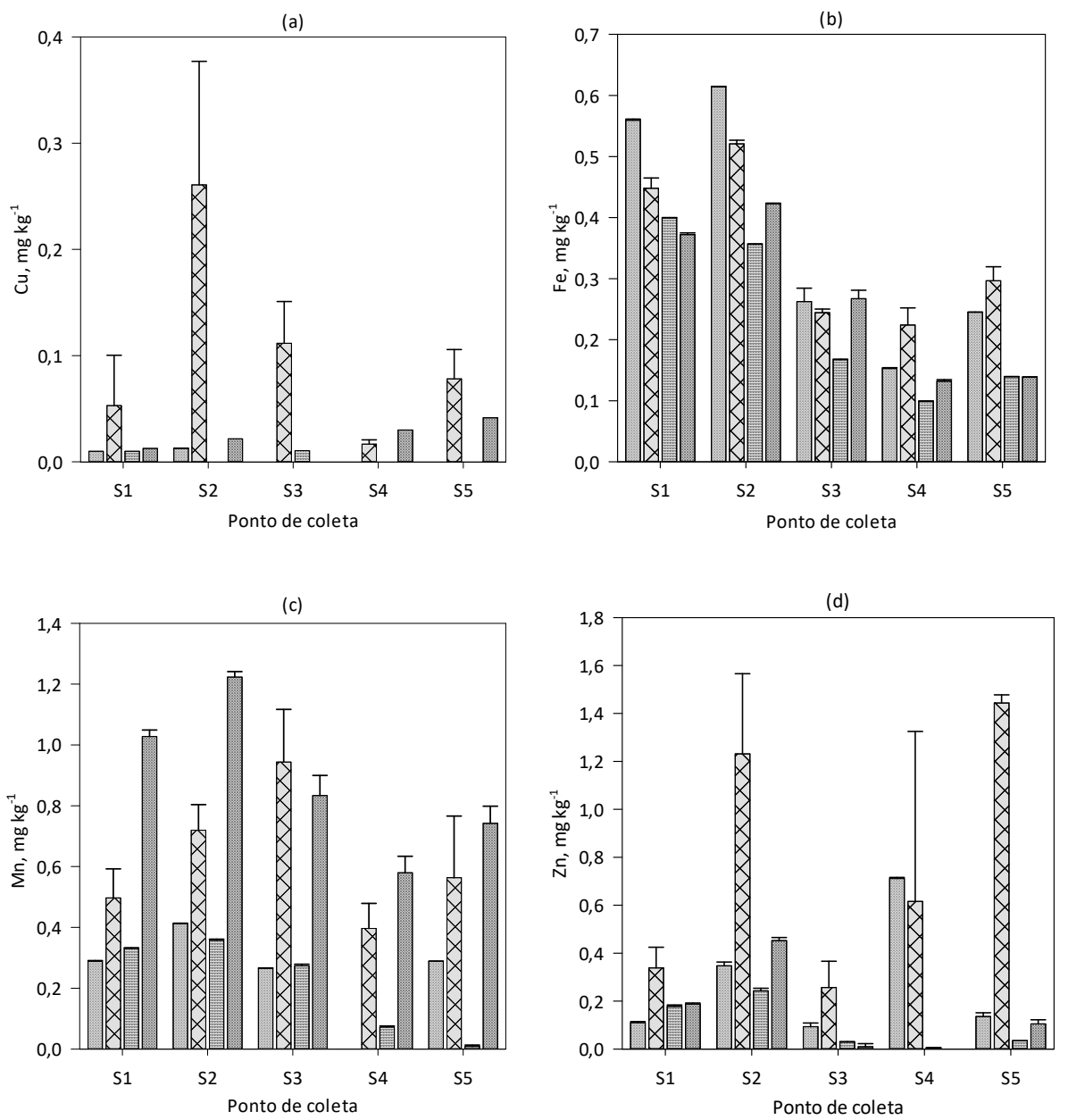

$\square$ 1'a coleta $\times$ 2a coleta $\square$ 3a coleta $\square$ 4a coleta

Figura 3: Concentração dos metais $\mathrm{Cu}(\mathrm{a}), \mathrm{Fe}(\mathrm{b}), \mathrm{Mn}(\mathrm{c})$ e $\mathrm{Zn}(\mathrm{d})$ nos sedimentos de fundo nos pontos de coleta S1, S2, S3, S4 e S5 na BHE nos meses de Março/19, Junho/19, Agosto/19 e Dezembro/19. 

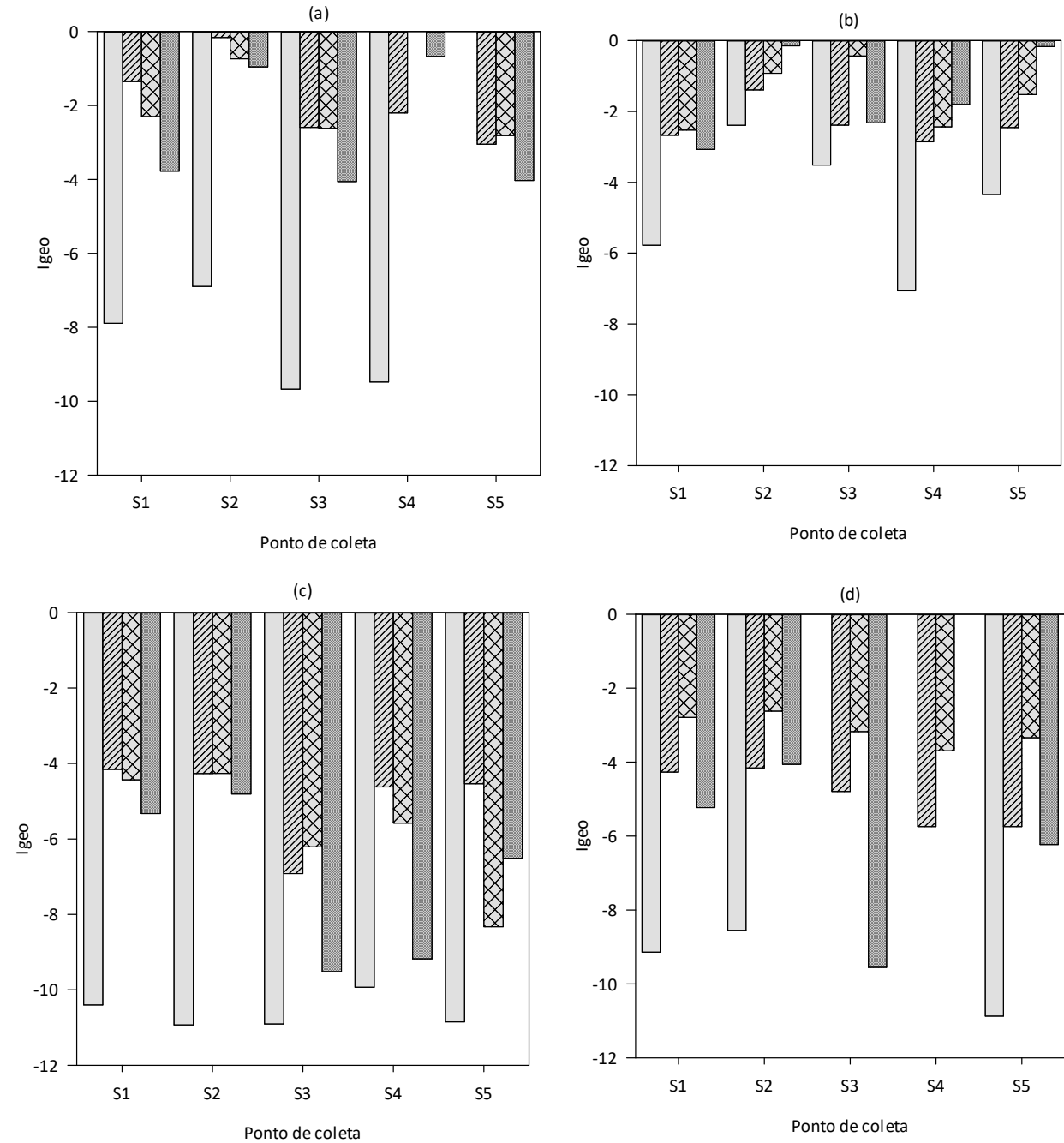

$\square \mathrm{Cu} \quad$ WIIA $\mathrm{Fe} \quad \square \mathrm{Mn} \quad \square \mathrm{Zn}$

Figura 4: Índice de Geoacumulação (Igeo) para os pontos de estudo (S1, S2, S3, S4 e S5) na Bacia Hidrográfica do Epaminondas para as coletas de Março (a), Junho (b), Agosto (c) e Dezembro (d) para os metais Cu, Fe, Mn e Zn.

O Fator de Enriquecimento (FE) para o $\mathrm{Cu}$, Fe e $\mathrm{Zn}$, indica que os teores observados nos sedimentos são referentes ao solo e ao ambiente natural, devido as concentrações observadas dos mesmos classificarem os mesmos em valores de FE até 1,5. Para o Mn ocorre indicativo que a fonte do metal é de origem antrópica, devido ao enquadramento do $\mathrm{Mn}$ no FE com valores acima de 1,5, esse mesmo processo ocorre para o Fator de Contaminação (Tabela 3).

Tabela 3: Valores dos Fatores de Enriquecimento (FE), Fator de Contaminação (FC), Índice de Carga Poluidora (ICP), Grau de Contaminação $(\mathrm{GC})$ e Grau de Contaminação modificado $(\mathrm{GCm})$ para sedimentos de fundo na Bacia Hidrográfica Epaminondas no período de estudo.

\begin{tabular}{|c|c|c|c|c|c|c|c|c|c|c|c|}
\hline \multirow{2}{*}{ Coleta } & \multicolumn{4}{|l|}{$\mathrm{FE}$} & \multicolumn{4}{|l|}{ FC } & \multirow{2}{*}{ ICP } & \multirow{2}{*}{$\mathrm{GC}$} & \multirow{2}{*}{$\mathrm{GCm}$} \\
\hline & $\mathrm{Cu}$ & $\mathrm{Fe}$ & $\mathrm{Mn}$ & $\mathrm{Zn}$ & $\mathrm{Cu}$ & $\mathrm{Fe}$ & $\mathrm{Mn}$ & $\mathrm{Zn}$ & & & \\
\hline & S1 & & & & & & & & & & \\
\hline $1 \underline{a}$ & 0,01 & 1,11 & 3,45 & 0,26 & 0,00 & 1,14 & 3,63 & 0,02 & 0,09 & 4,78 & 1,20 \\
\hline $2^{a}$ & 0,04 & 0,27 & 1,82 & 0,27 & 0,00 & 0,46 & 3,10 & 0,03 & 0,11 & 3,59 & 0,90 \\
\hline $3 \underline{a}$ & 0,00 & 0,03 & 0,16 & 0,02 & 0,00 & 0,16 & 0,83 & 0,01 & 0,02 & 0,99 & 0,25 \\
\hline \multirow[t]{2}{*}{$4 \underline{a}$} & 0,01 & 0,15 & 2,58 & 0,10 & 0,00 & 0,15 & 2,58 & 0,01 & 0,03 & 2,73 & 0,68 \\
\hline & S2 & & & & & & & & & & \\
\hline $1 \underline{a}$ & 0,01 & 0,24 & 3,62 & 0,43 & 0,00 & 0,53 & 5,15 & 0,05 & 0,12 & 5,73 & 1,43 \\
\hline $2^{\mathrm{a}}$ & 0,14 & 0,25 & 2,05 & 0,66 & 0,02 & 0,15 & 4,50 & 0,09 & 0,18 & 4,76 & 1,19 \\
\hline $3 \underline{a}$ & 0,00 & 0,14 & 0,82 & 0,11 & 0,00 & 0,17 & 0,89 & 0,01 & 0,02 & 1,07 & 0,27 \\
\hline 4 & 0,01 & 0,18 & 3,05 & 0,21 & 0,00 & 0,53 & 3,05 & 0,01 & 0,06 & 3,59 & 0,90 \\
\hline
\end{tabular}




\begin{tabular}{|c|c|c|c|c|c|c|c|c|c|c|c|}
\hline $1 \underline{a}$ & 0,01 & 4,74 & 3,62 & 0,28 & 0,00 & 0,25 & 3,33 & 0,01 & 0,04 & 3,59 & 0,90 \\
\hline $2 \underline{a}$ & 0,06 & 0,11 & 2,51 & 0,13 & 0,01 & 0,25 & 5,90 & 0,02 & 0,12 & 6,18 & 1,54 \\
\hline $3 \underline{a}$ & 0,01 & 0,19 & 1,85 & 0,04 & 0,00 & 0,07 & 0,68 & 0,00 & 0,01 & 0,75 & 0,19 \\
\hline \multirow[t]{2}{*}{$4 \underline{a}$} & 0,00 & 0,11 & 2,08 & 0,00 & 0,00 & 0,11 & 2,08 & 0,00 & 0,00 & 2,19 & 0,55 \\
\hline & S4 & & & & & & & & & & \\
\hline $1^{\mathrm{a}}$ & 0,00 & 0,37 & 0,00 & 1,40 & 0,00 & 0,50 & 0,00 & 0,11 & 0,00 & 0,61 & 0,15 \\
\hline $2^{\mathrm{a}}$ & 0,01 & 0,17 & 1,77 & 0,52 & 0,00 & 0,23 & 2,48 & 0,05 & 0,07 & 2,75 & 0,69 \\
\hline $3 \underline{a}$ & 0,00 & 0,02 & 0,08 & 0,00 & 0,00 & 0,04 & 0,18 & 0,00 & 0,00 & 0,22 & 0,05 \\
\hline \multirow[t]{2}{*}{$4 \underline{a}$} & 0,00 & 0,05 & 1,45 & 0,00 & 0,00 & 0,05 & 1,45 & 0,00 & 0,00 & 1,50 & 0,38 \\
\hline & S5 & & & & & & & & & & \\
\hline $1^{\mathrm{a}}$ & 0,00 & 0,67 & 4,86 & 0,44 & 0,00 & 0,50 & 3,60 & 0,02 & 0,00 & 4,12 & 1,03 \\
\hline $2 \underline{a}$ & 0,05 & 0,17 & 1,91 & 0,92 & 0,01 & 0,30 & 3,53 & 0,11 & 0,16 & 3,94 & 0,99 \\
\hline $3 \underline{a}$ & 0,00 & 0,03 & 0,01 & 0,01 & 0,00 & 0,06 & 0,03 & 0,00 & 0,00 & 0,08 & 0,02 \\
\hline $4 \underline{a}$ & 0,00 & 0,06 & 1,85 & 0,05 & 0,00 & 0,06 & 1,85 & 0,00 & 0,01 & 1,91 & 0,48 \\
\hline
\end{tabular}

1a coleta: Março/2019; 2a coleta: Junho/2019; 3a coleta: Agosto/2019; 4a coleta: Dezembro/2019

\section{DISCUSSÃO}

Os teores de $\mathrm{Cu}$ encontrados corroboram com alguns trabalhos (XIAO et al., 2019; SHI et al., 2019) nos quais, sua concentração sempre é menor que a do Zn. Segundo Santos et al. (2017) os teores de Cu podem apresentarem-se em menores concentrações em áreas que a granulometria dos sedimentos, encontra-se na fração areia, devido ao tamanho da partícula do sedimento. Os mesmos autores observaram que em áreas que a fração areia grossa predominava nos sedimentos as concentrações de metais pesados eram superiores as áreas com areia fina. Isso pode ser explicado levando em consideração que o $\mathrm{Cu}$ apresente preferência de adsorção pela matéria orgânica e o Zn pela fração mineral, onde essa fração é mais facilmente perdida em processos erosivos que originam os sedimentos (CADONÁ et al., 2019).

Para os teores de Fe e Mn mais elevados quando comparados aos teores de $\mathrm{Cu}$ e $\mathrm{Zn}$, devido ao fato que a bacia apresenta problemática de elevação desses elementos nas águas da barragem do Santa Bárbara, fato este comprovado em estudo realizado por Kath et al. (2017), onde os autores explicam que no ano de 2007, a Barragem do Santa Bárbara apresentou problemas para o abastecimento humano do município de Pelotas/RS, relacionado às quantidades excessivas de $\mathrm{Mn}$ dissolvido na água. Para esse fenômeno de $\mathrm{Mn}$ dissolvido elevado na água, os autores encontraram relação positiva, entre os teores de $\mathrm{Mn}$ e $\mathrm{P}$ total, que podem estar associados a ausência de coleta e tratamento de efluentes e resíduos domésticos, bem como, a erosão de partículas de solo com $\mathrm{P}$ adsorvido que é carreado aos corpos hídricos em eventos pluviométricos.

Chen et al. (2015) ao avaliarem o lgeo para os metais $\mathrm{Cr}$, Cu, As, Ni, Pb e Zn também observaram que, a aplicação do Igeo resultou em classe 1, ou seja, com nula ou baixa contaminação. Neste mesmo local de estudo já foi observado altos teores de Fe e Mn dissolvido na água (KATH et al., 2017) e possivelmente grande parte dessa alta concentração por estar associada a grande produção de sedimentos ao longo da bacia causado por processos de erosão do solo. Outro fator importante na elevação ou diminuição das concentrações de metais na água deve-se pela presença de matéria orgânica (MO), a qual é responsável pela complexação e quelação desses metais, aumentando ou reduzindo a disponibilidade desses em suas frações dissolvidas (CADONÁ et al., 2019). Apesar do Igeo indicar que não ocorre contaminação por esses metais nos sedimentos, os teores de Fe e Mn se encontram mais próximos a 0 ou a 1, quando comparados ao Cu e Zn. 
De acordo com Xiao et al. (2019), o Mn é o metal com maior tendência de contaminação nos sedimentos, quando comparado ao $\mathrm{Cu}$ e $\mathrm{Zn}$.

A origem antrópica do $\mathrm{Mn}$ pode ser melhor entendida ao avaliar o estudo realizado por Kath et al. (2017), onde os autores avaliaram a relação do Mn dissolvido com características físico-químicas da água na Barragem do Santa Bárbara e observaram que uma das possíveis razões para os altos teores do Mn dissolvido na água, é a interação desse elemento com o P total, devido a conhecida relação entre os dois em áreas de solos hidromórficos, como ocorre na porção sul da bacia, onde há predomínio de Planossolos e Gleissolos (CUNHA et al., 1996). Caracteristicamente Planossolos e Gleissolos ocorrem em porções mais baixas da paisagem, próximas as fontes de água, passiveis de alagamento e oscilação do lençol freático em curtos intervalos de tempo, o que favorece a presença de concreções de Mn nos horizontes do solo. Dessa forma, os valores indicativos de contaminação por $\mathrm{Mn}$ para os sedimentos de fundo, podem estar associados aos teores de P disponível ou total nos sedimentos, bem como, é pertinente citar, que a erosão na bacia vem ocorrendo nas camadas superficiais do solo, as quais, apresentam os maiores teores de P disponível.

Como relação ao Índice de Carga Poluidora, Grau de Contaminação e Grau de Contaminação modificado (Tabela 5) se observou que não há indícios de contaminação e risco ambiental de contaminação pelos metais em estudo, apesar dos demais índices indicarem processo de contaminação pelo $\mathrm{Mn}$. O cenário demostrado pela análise dos dados da Tabela 5 indica que o processo de contaminação pode estar associado a baixa concentração encontrada dos metais nos sedimentos de fundo (Figura 12) e pelo fato de que a bacia ter predomínio de uso agrícola, sem aporte de efluentes industriais, que afetam sensivelmente os teores desses metais, como ocorre em diferentes regiões do Brasil (TORREZANI et al., 2016; NASCIMENTO et al., 2018).

\section{CONCLUSÕES}

A aplicação do Índice de Geoacumulação e seus índices associados, indicaram que os sedimentos de fundo na Bacia Hidrográfica do Epaminondas não estão contaminados por $\mathrm{Cu}, \mathrm{Fe}, \mathrm{Mn}$ e $\mathrm{Zn}$ pelos metais em estudo para todos os pontos e os períodos de estudo e a aplicação do Fator de Enriquecimento indicou que todos os metais são oriundos do solo. No entanto, mesmo não ocorrendo indicativo de contaminação, ocorre a problemática do Fe e Mn na barragem do Santa Bárbara, necessitando de estudo contínuos e sequenciais desses elementos, bem como ações para o controle dos processos erosivos do solo, para diminuir o aporte de sedimentos nos corpos hídricos.

\section{REFERÊNCIAS}

ABRAHIM, G. M. S.; PARKER, R. J.. Assessment of heavy metals enrichment factors and the degree of contamination in marine sediments from Tamaki Estuary, Auckland, New Zealand. Environmental Monitoring Assessment., Atlanta, v.136, n.1-3, p.227-238, 2008. DOI: https://doi.org/10.1007/s10661-007-9678-2

CADONÁ, E. A.; WILBERT, G. F.; SANTOS, M. L.; SOARES, C. R. F. S.; LOSS, A.; SOUZA, E. L.; LOURENZI, C. R.. Cu, Zn e Mn na água e no solo em áreas com intense atividade suinícola no Sudeste de Santa Catarina. In: PACHECO, J. T. R.; KAWANISHI, J. Y.; NASCIMENTO, R.. Meio Ambiente e Desenvolvimento Sustentável 2. Ponta Grossa: Atena, 2019. p.258-270.

CHEN, H.; TENG, Y.; LU, S.; WANG, Y.; WANG, J. Contamination features and health risk of soil heavy metals in China. Science of the Total Environment, Barcelona, 
v.512-513, p.143-153, 2015. DOI: https://doiorg.ez66.periodicos.capes.gov.br/10.1016/i.scitotenv.2015.0 1.025

CUNHA, N. G.; SILVEIRA, J. C.. Levantamento de solos do município de Pelotas. Pelotas: EMBRAPA, 1996.

GONÇALVES, P. E. R. S.; OLIVEIRA, A. P.; CRUZ, I. F.; ZEILHOFER, P.; DORES, E. F. G. C.. Distribuição espacial de metais potencialmente tóxicos em água superficial nas bacias dos rios Cuiabá e São Lourenço - MT. Revista Brasileira de Recursos Hídricos, Porto Alegre, v.20, n.1, p.157-168, 2015. DOI:

http://dx.doi.org/10.21168/rbrh.v20n1.p157-168

KATH, A. H.; TIMM, J. G.; MONKS, J. L. F.. Caracterização de parâmetro físico-químicos e correlações com o manganês nos afluentes da barragem do Santa Bárbara, Pelotas/RS.

Revista Ibero-Americana de Ciências Ambientais, Aracaju, v.8, n.2, p.34-48, 2017. DOI:

https://doi.org/10.6008/SPC2179-6858.2017.002.0003

KLUMB, G. B.; VEBER, P. M.; MELLO, L. B.; DORNELES, V. R.; DAMÉ, R. C. F.; TEIXEIRA-GANDRA, C. F. A.. Balanço hídrico climatológico e classificação climática de Thornthwaite para o município de Pelotas/RS. Brazilian Journal of Animal and Environmental Research, Curitiba, v.1, n.1, p.229-235, 2018.

KORASHY, H. M.; ATTAFI, I. M.; FAMULSKI, K. S.; BAKHEET, S. A.; HAFEZ, M. M.; ALSAAD, A. M. S.; AL-GHADEER, A. R. M.. Gene expression profiling to identify the toxicities and potentially relevant human disease outcomes associated with environmental heavy metal exposure. Environmental Pollution, Amã, v.221, p.64-74, 2017. DOI: https://doi.org/10.1016/j.envpol.2016.10.058

MATOS, L. A.; CUNHA, A. C. S.; SOUSA, A. A.; MARANHÃO, J. P. R.; SANTOS, N. R. S.; GONCALVES, M. M. C.; DANTAS, S. M. M. M.; SOUSA, J. M. C.; PERON, A. P.; SILVA, F. C.; ALENCAR, M. V. O. B.; ISLAM, M. T.; AGUIAR, R. P. S.; MELO-

CAVALCANTE, A. A. C.; BONECKER, C. C.; JUNIOR, H. F. J.. The influence of heavy metals on toxicogenetic damage in Brazilian Tropical River. Chemosphere, v.185, p.852-859, 2017. DOI: https://doi-

org.ez66.periodicos.capes.gov.br/10.1016/j.chemosphere.20 17.07.103

MÜLLER, G.. Schwermetalle in den sedimenten des RheinsVeränderungen seit 1971. Umschan, v.79, p.778-783, 1979.

NASCIMENTO, L. P.; REIS, D. A.; ROESER, H. M. P.; SANTIAGO, A. F.. Avaliação geoquímica de metais em sistemas fluviais afetados por atividades antrópicas no Quadrilátero Ferrífero.
Engenharia Sanitária e Ambiental, Rio de Janeiro, v.23, n.4, 2018, p.767-778. Dol: https://doi.org/10.1590/s141341522018165852

OLIVEIRA, E. C.; LAFON, J. M.; CORRÊA, J. A. M.; CARVALHO, J. H. S.; DIAS, F. F.; TADELI, M. H. T.. Distribuição dos metais traços em sedimentos de fundo do sistema hidrográfico da região de Belém, PA (margem oeste da baía do Guarajá e do rio Carnapijó). Geochemica Brasiliensis, v.29, p.139-153, 2015. DOI: http://dx.doi.org/10.21715/GB23582812.2015292139

SANTOS, L. M. M.; SOUZA, R. C.; ANUNCIAÇÃO, D. S.; VIANA, Z. C. V.; SANTOS, V. L. C. S.. Análise de metais em sedimentos de manguezal do Rio Passa Vaca, Salvador, Brasil. Acta Brasiliensis, v.1, p.1-7, 2017. DOI: https://doi.org/10.22571/Actabra12201

SHI, C.; DING, H.; ZAN, Q.; LI, R.. Spatial variation and ecological risk assessment of heavy metals in mangrove sediments across China. Marine Pollution Buletin, v.143, p.115-124, 2019. DOI: https://doiorg.ez66.periodicos.capes.gov.br/10.1016/j.marpolbul.2019. $\underline{04.043}$

SILVA, F. C.. Digestão nitroperclórica: manual de análises química de solos, plantas e fertilizantes. Brasília: EMBRAPA, 1999.

TEIXEIRA, P. C.; DONAGEMMA, G. K.; FONTANA, A. TEIXEIRA, W. G.. Manual de Métodos de Análise de Solo. 3 ed. Brasília: EMBRAPA, 2017.

TORREZANI, L.; SARGENTINI JUNIOR, É.; OLIVEIRA, C. A. S.; SANTANA, G. P.. Índice de Geoacumulação de Mercúrio na Bacia do Igarapé do Educandos (Manaus/Amazonas). Journal of Chemical Engineering and Chemistry, v.2, n.3, p.161-170, 2017. DOI: https://doi.org/10.18540/jcecvl2iss3pp161-170

YOUSSEF, M.; EL-SOROGY, A.; OSMAN, M.; GHANDOUR, I.; MANAA, A.. Distribution and metal contamination in core sediments from the Al-Wajh area, Red Sea, Saudi Arabia. Marine Pollution Bulletin, v.152, p.1-9, 2020. DOI: https://doi-

org.ez66.periodicos.capes.gov.br/10.1016/j.marpolbul.2020. $\underline{110924}$

XIAO, H.; SHAHAB, A.; LI, J.; XI, B.; SUN, X.; HE, H.; YU, G. Distribution, ecological risk assessment and source identification of heavy metals in surface sediments of Huixian karst wetland, China. Ecotoxicology and Environment Safety, v.185, p.1-10, 2019. DOI: https://doi.org/10.1016/j.ecoenv.2019.109700

A CBPC - Companhia Brasileira de Produção Científica (CNPJ: 11.221.422/0001-03) detém os direitos materiais desta publicação. Os direitos referem-se à publicação do trabalho em qualquer parte do mundo, incluindo os direitos às renovações, expansões e disseminações da contribuição, bem como outros direitos subsidiários. Todos os trabalhos publicados eletronicamente poderão posteriormente ser publicados em coletâneas impressas sob coordenação da Sustenere Publishing, da Companhia Brasileira de Produção Científica e seus parceiros autorizados. Os (as) autores (as) preservam os direitos autorais, mas não têm permissão para a publicação da contribuição em outro meio, impresso ou digital, em português ou em tradução. 